Repr. Nutr. Dévelop., 1986, 26, (3), 863-875.

\title{
Variations saisonnières du diamètre testiculaire et de la morphologie des spermatozoïdes chez le bélier Vendéen et chez le bélier Texel
}

\author{
G. COLAS, Y. GUÉRIN, Y. LEMAIRE $(*), Y$. MONTASSIER $\left({ }^{* *}\right)$, J. DES- \\ PIERRES $(*)$
}

Station de Physiologie de la Reproduction, I.N.R.A., Nouzilly, 37380 Monnaie, France.

(*) U.P.R.A., Ile-de-France, 02400 Château-Thierry,

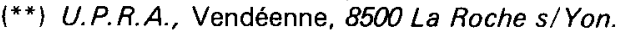

Summary. Seasonal variations in the testicular diameter and sperm morphology of Vendean and Texel rams.

Nineteen Vendean ( $V$ ) rams (7 yearlings about 15 months old and 12 adults of $>2$ years) and 13 Texel (T) adult ( $>2$ years) rams were controlled for body weight (BW), testis diameter (TD) and sperm morphology for two consecutive years (1982 and 1983) from February to June (T) or July (V) and from September to November the first year, from February to June and from September to October the second year.

BW was recorded every month. TD was measured once weekly using a caliper. The percentage of abnormal spermatozoa (MA) was determined on each ejaculate (1 $\mathrm{ej} / \mathrm{ram} /$ week) as described previously (Colas, 1980).

BW increased rapidly $(P<0.01)$ in the T rams the first 4 months and did not augment significantly any more afterwards. In the $\mathrm{V}$ breed, no significant variations appeared at any time of the experiment, except in the last month when BW decreased $(P<0.05)$. However, in the yearlings (V), there was considerable body growth between February and October 1982 but no significant growth afterwards. In the adults, no statistical variations occurred at any time of the experimental period.

TD varied greatly during the year in the $V$ rams. During both years, it was minimal in May $(59.0 \pm 5.7 \mathrm{~m} / \mathrm{m}$ in $1982 ; 58.1 \pm 4.4 \mathrm{~m} / \mathrm{m}$ in 1983); in September it was already regressing.

There were also large seasonal variations in the TD profile of the T breed but its curve was different from one year to another : the mean value was minimal in February 1982 $59.5 \pm 6.0 \mathrm{~m} / \mathrm{m})$ and from February $(67.5 \pm 4.4 \mathrm{~m} / \mathrm{m})$ to May $(68.6 \pm 5.0 \mathrm{~m} / \mathrm{m}) 1983$.

In both breeds, testicular growth always started in June, i.e. during the long days, whatever the year.

The importance of the variations and their time of onset during the year suggest that both breeds were very sensitive to photoperiod. These results also permit a generalization of the idea that testicular activity in male sheep recommences in the long days.

Both breeds showed large individual variations between the minimal values recorded in spring and the highest ones measured in September 12.7 to $78.0 \%$ and 2 to $100 \%$, respectively, for $V$ and $T$ rams in $1982 ; 5.5$ to $100 \%$ and 14 to $84 \%$ in 1983 ).

On the whole, MA were more frequent from February to June than from September to October or November in both breeds.

In the $V$ breed, MA were highest in April $135.1 \%$ in 1982, $33.1 \%$ in 1983). In the T breed, maximal mean values were lower the first year than the second year $129.8 \%$ in 
1982, $38.5 \%$ in 1983) and appeared at different periods (beginning of March in 1982, end of May in 1983).

Both breeds showed considerable individual differences in MA in spring $1+5.2$ to $+21.9 \%$ and +15.8 to $+48.7 \%$, respectively, for $V$ and $T$ rams in $1982 ;+20.0$ to

$+39.6 \%$ and +15.2 to $+31.8 \%$ in 1983). In autumn, these differences were much less.

MA were mainly composed of head abnormalities, especially from February to June (V : $r=+0.885, T: 0.856 ; P<0.01)$.

This study shows that sexual activity and sperm quality exhibit large seasonal variations which depend on the individual.

\section{Introduction}

Chez le bélier lle-de-France, la fonction spermatogénétique et la qualité des gamètes subissent, sous l'effet des variations quotidiennes de la durée d'éclairement, de profondes modifications au cours de l'année (Ortavant et Thibault, 1956; Ortavant, 1958 ; Pelletier, 1971; Albério, 1976 ; Colas, 1980, 1981). D'autres races, la race Vendéenne et la race Texel en particulier, sont connues pour leur faible activité sexuelle au printemps mais, jusqu'à présent, aucune étude précise ne leur a été consacrée. Pour bien connaître les aptitudes de reproduction de ces deux génotypes très utilisés en élevage ovin, nous avons procédé à deux types de mesures : celle du diamètre antério-postérieur du testicule, très lié à la production spermatique (Colyer, 1971; Courot, 1971 ; Albério, 1976) et celle du pourcentage de spermatozoïdes anormaux, dont dépend, au moins partiellement, le pouvoir fécondant de l'éjaculat chez le bélier (Wiggins, Terrill and Emik 1953 : Colas, 1981).

Cette analyse doit nous permettre de savoir :

1. S'il existe des variations saisonnières dans la taille de la gonade et dans la qualité du sperme et si ces variations se répètent d'une année à l'autre.

2. $\mathrm{Si}$, à l'intérieur d'une même race, on peut décéler, comme chez l'lle-deFrance, des différences individuelles importantes.

La connaissance de ces données est importante dans la mesure où elle devra déterminer le choix et l'utilisation des géniteurs au printemps et améliorer ainsi le succès de la reproduction à cette saison.

\section{Matériel et méthodes}

I. Animaux expérimentaux. - Cette étude a été réalisée pendant deux années consécutives (1982 et 1983) sur les races Texel (T) et Vendéennes (V) dans leur lieu de sélection (Station de Testage).

19 béliers $V$ ( 7 antenais d'environ 15 mois) et 12 adultes (de plus de 2 ans) et 13 béliers $T$ ( $>2$ ans) ont été utilisés la première année. 26 d'entre eux (14 V et $12 \mathrm{~T}$ ) ont subi les mêmes contrôles pendant une année supplémentaire.

Les mâles vivaient dans une bergerie et avaient accès, lorsque les conditions climatiques le permettaient, à un pré clôturé. 
II. Contrôles. - Les collectes de sperme et les contrôles (pesée, diamètre testiculaire, anomalies morphologiques) ont eu lieu en 1982, de février à juin (T) ou juillet (V) et de septembre à novembre. L'année suivante ils ont été réalisés à peu près aux mêmes périodes mais, pour des raisons purement matérielles, certains d'entre eux n'ont pu être effectués.

a. Pesée ( $P C$ ). - Les animaux ont été pesés (à $0,5 \mathrm{~kg}$ près) une fois par mois pendant toute la durée de l'expérience.

b. Diamètre testiculaire (DT). - Chaque semaine, le diamètre antéropostérieur maximum des deux testicules a été mesuré à l'aide d'un pied à coulisse. Ces mesures ont toujours été réalisées, pour chaque race, par le même opérateur.

c. Anomalies morphologiques (AM). - Les éjaculats ont été collectés à l'aide d'un vagin artificiel une fois par semaine 11 seul prélèvement par séance et par animal) en présence d'une femelle non en chaleur.

La préparation de la semence en vue de son contrôle morphologique et de l'identification des anomalies a été effectuée selon la méthode habituelle du laboratoire (Colas, 1980) : les frottis ont été confectionnés à partir de sperme prédilué dans une solution de lactose-jaune d'œuf (Nagase et Graham, 1964). Le nombre et la nature des malformations cellulaires ont été déterminés sur un total de 150 spermatozoïdes.

III. Expression des résultats. - L'évolution des données (PC et DT) en fonction de la saison est exprimée sous forme de graphiques à partir des moyennes hebdomadaires ( \pm leur écart-type) calculées sur l'ensemble des individus de chaque race.

En plus du pourcentage total d'anomalies morphologiques, nous avons calculé la proportion de spermatozoïdes porteurs de malformations de la tête (lésions de l'acrosome, micro- ou macro-céphalies, têtes pyriformes ou allongées etc...), connues pour leur effet sur la fécondance (Colas, 1981) et de gouttelettes cytoplasmiques proximales qui traduisent généralement une mauvaise maturation épididymaire (Fournier-Delpech et al., 1979).

Les moyennes hebdomadaires (PC, DT) ont été comparées par analyse de variance.

Les coefficients de corrélation entre anomalies de tête et anomalies totales ont été déterminés à partir des pourcentages moyens hebdomadaires calculés sur la totalité des animaux présents chaque année.

\section{Résultats}

\section{Evolution corporelle}

Dans l'ensemble (fig. 1), les béliers $V$ sont plus lourds que les béliers $T$, bien que leur effectif soit composé en partie d'animaux antenais, donc plus jeunes. C'est ce qui explique que l'hétérogénéité des poids corporels, exprimée par leur écart type, soit plus forte la première année chez les $V$ que chez les $T$. La seconde année, les écarts types sont du même ordre dans les deux races puisque les individus sont tous des sujets adultes. 


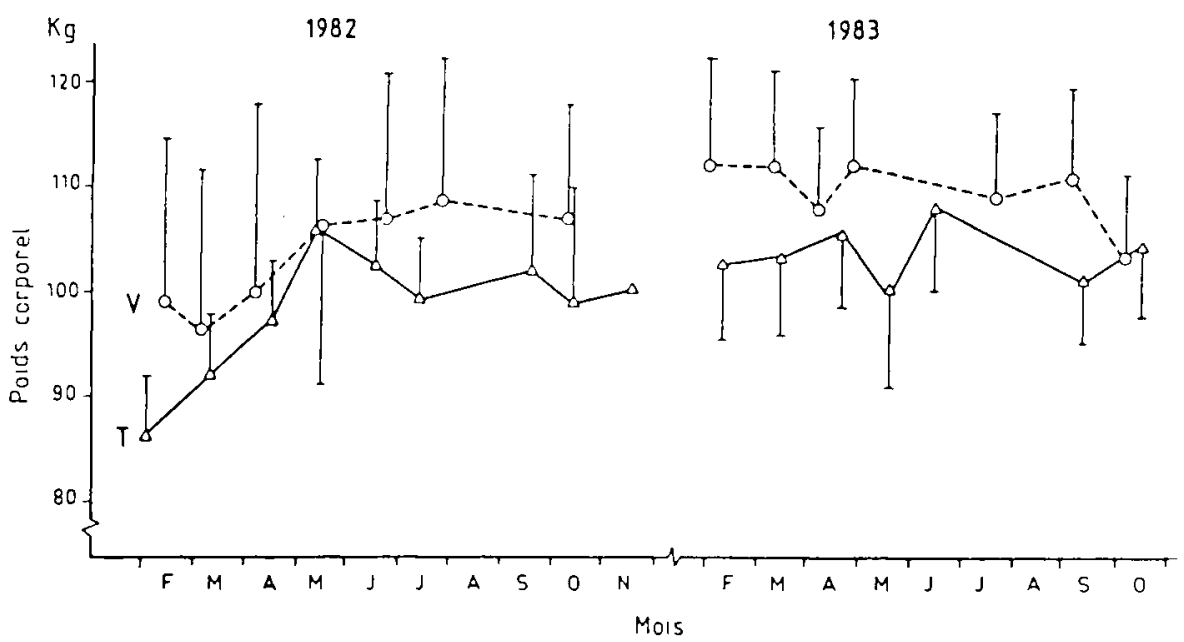

FIG. 1. - Evolution mensuelle du poids corpore/ (X $\pm \mathrm{DS})$ chez les béliers Vendéens (V) et chez les béliers Texel (T).

On voit également que le poids moyen des béliers $T$ augmente rapidement la première année entre les mois de février et mai (respectivement $86,6 \pm 6,1$ et $106,3 \pm 6,6 \mathrm{~kg} ; P<0,01)$. Au-delà, les valeurs ne diffèrent plus significativement.

Les données enregistrées en 1982 sur l'ensemble des béliers Vendéens entre les mois de février et mai ne sont pas différentes. Leur évolution ultérieure reste faible sauf en fin d'expérience (octobre) où leur moyenne a tendance à baisser. Toutefois, si on étudie séparément l'évolution corporelle des jeunes et des adultes, on constate que, chez ces derniers, le poids moyen reste à peu près le même pendant toute la période expérimentale alors que chez les antenais, la croissance est importante entre février et octobre 1982 (respectivement $80,0 \pm 3,7$ et $102,4 \pm 5,6 \mathrm{~kg} ; P<0,01)$ et s'arrête ensuite.

\section{Diamètre testiculaire (DT)}

Le DT varie beaucoup au cours de l'année chez la race Vendéenne (fig 2). Les moyennes mensuelles diminuent de février à la première quinzaine de juin en $1982(69,7 \pm 6,5$ vs $59,2 \pm 5,7 \mathrm{~mm} ; \mathrm{P}<0,01)$ et de février à mai en 1983 $(65,7 \pm 3,8$ vs $58,1 \pm 4,4 \mathrm{~mm} ; P<0,01)$, puis augmentent rapidement. Elles passent par un maximum sans doute au mois d'août puisqu'en 1982 et 1983 elles régressent dès les premiers jours de septembre.

Le DT subit la même évolution chez l'Antenais et chez l'adulte (fig. 3) mais les niveaux atteints sont évidemment plus faibles chez le jeune.

Les variations relatives du DT, calculées entre les valeurs minimales et maximales sont très différentes selon les béliers : $+21,9$ à $+5,2 \%$ en $1982,+20,0$ à $+39,6 \%$ en 1983 . 
Chez le bélier Texel, on note aussi de grandes fluctuations dans les dimensions testiculaires au fil des saisons (fig. 2). Toutefois, l'évolution des données est assez dissemblable d'une année à l'autre. Ainsi, la moyenne du mois de février 1982 est inférieure $(P<0,01)$ à celle des trois mois suivants alors qu'en 1983, les valeurs sont assez stables entre février et mai et ne diffèrent pas significativement. De même, la régression testiculaire observée dès le mois de septembre la première année, n'est pas encore amorcée le 15 octobre l'année suivante. Il semble, en revanche, que la croissance de la gonade ait toujours lieu en juin comme en témoignent les derniers contrôles hebdomadaires du printemps $(66,0 \pm 4,3$ et $70,1 \pm 5,4 ; P<0,01$; respectivement les 1 et $22.6 .82 ; 70,4 \pm 4,4$ et $73,3 \pm 5,7$ les 7 et 14.6.83). En 1983, I'augmentation du DT n'est pas significative mais elle apparaît chez tous les animaux sauf un.

II existe aussi une variabilité importante dans les écarts entre DT minimal et maximal : de 15,8 à $48,7 \%$ en 1982 , de 15,2 à $31,8 \%$ en 1983 .

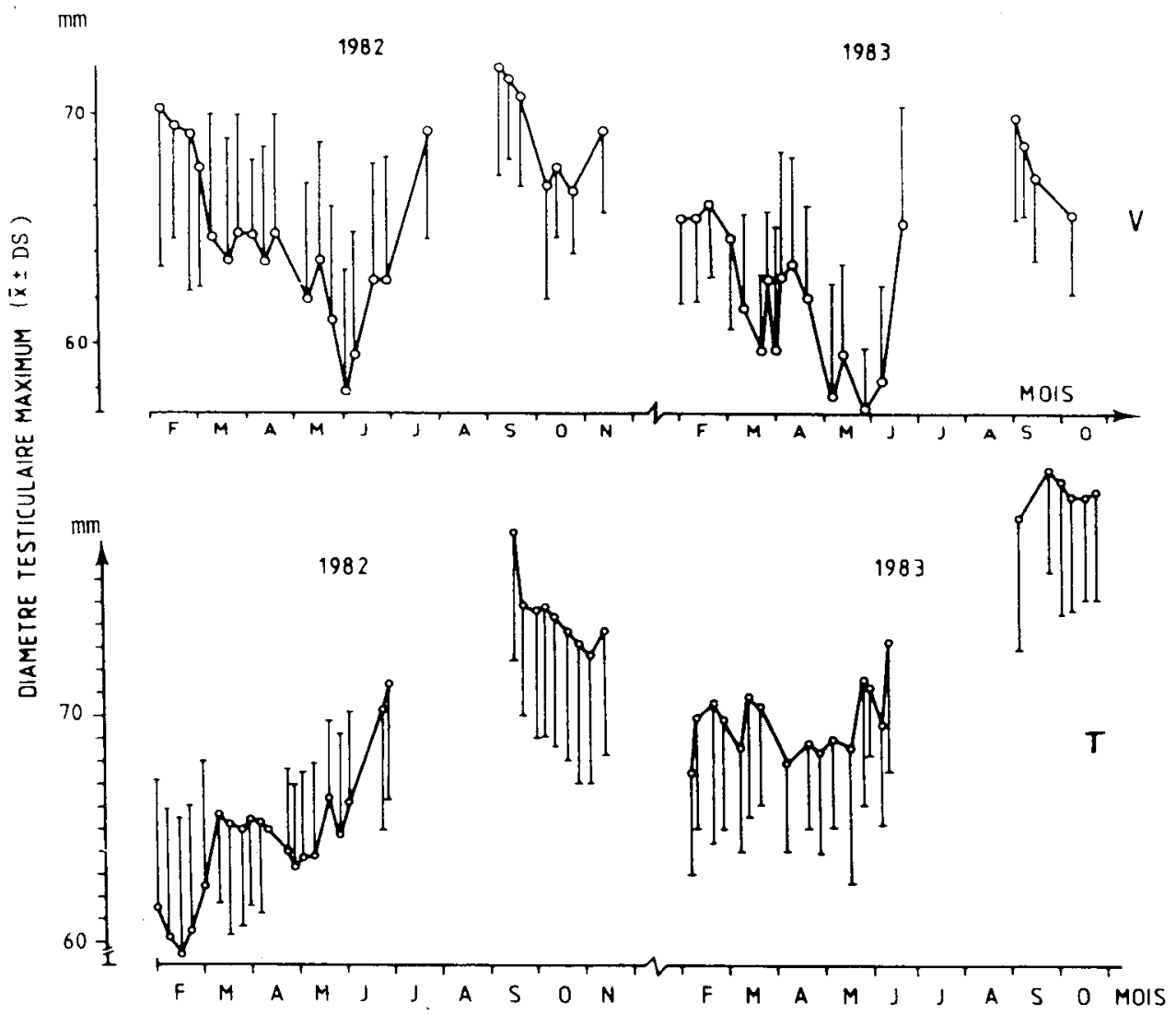

FIG. 2. - Evolution hebdomadaire du diamètre testiculaire chez le bélier Vendéen (V) et chez le bélier Texel (T) pendant deux années consécutives. 


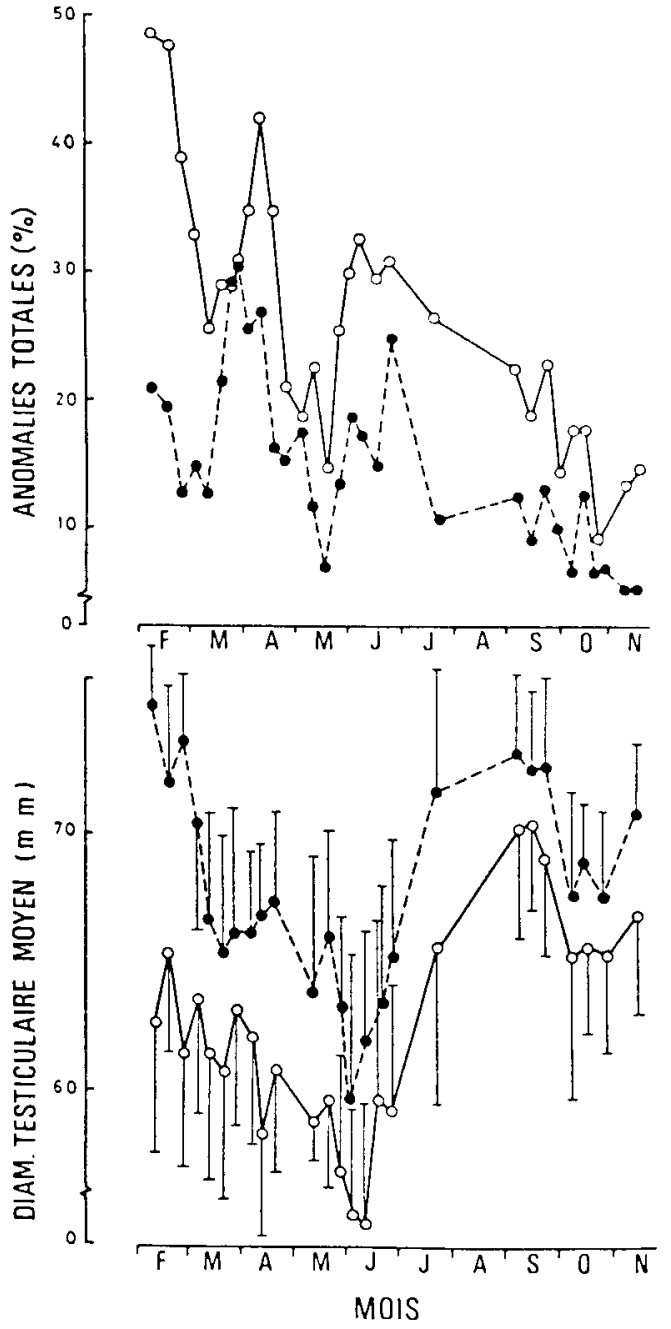

FIG. 3. - Evolution hebdomadaire du diamètre testiculaire et du pourcentage d'anomalies morphologiques chez le bélier Vendéen antenais $\left(\circ \_-0\right)$ et adulte (•——).

\section{Anomalies morphologiques}

Le pourcentage de spermatozoïdes anormaux est en général plus élevé, dans les deux races, de février à juin que de septembre à octobre ou novembre (fig. 4 et 5).

Chez les béliers Vendéens, c'est au mois d'avril que l'on observe le plus d'anomalies cellulaires [35,1\% en 1982 (valeurs extrêmes : 2,7 et 78,0 ), $33,9 \%$ en 1983 (valeurs extrêmes : 5,5 et $100 \%$ )]. Celles-ci diminuent ensuite assez rapidement pour augmenter à nouveau en juin. Chez les mâles de race Texel les maxima sont moins élevés la première année que la seconde année $[29,8 \%$ de spermatozoïdes anormaux en 1982 (valeurs extrêmes $2 \%$ et $100 \%$ ) vs $38,5 \%$ en 
1983 (valeurs extrêmes : $14 \%$ à $84 \%$ )] et s'observent à des périodes différentes (début mars en 1982, début mai en 1983).

Le sperme des béliers antenais (V) suit une évolution morphologique comparable à celle des adultes mais les anomalies $y$ sont beaucoup plus nombreuses (fig. 3). II faut attendre la fin du mois d'octobre pour trouver dans les deux lots des valeurs voisines.

Dans les deux races, on relève d'énormes différences individuelles dans l'évolution du profil morphologique des spermatozoïdes au cours de l'année (fig. 6).

Les anomalies de la tête des spermatozoïdes évoluent comme l'ensemble des malformations cellulaires. Les corrélations sont hautement significatives au printemps (tabl. 1) quels que soient la race, l'âge des animaux et l'année. De septembre à octobre ou novembre, elles ne sont significatives que dans la race Vendéenne.

Les gouttelettes cytoplasmiques proximales n'apparaissent que pendant la période des jours croissants chez les béliers $\mathrm{V}$ (fig. 4). Leur pourcentage est maximum en avril $[2,4 \%$ en $1982(0$ à $28 \%), 2,7 \%$ en $1983(0$ à $3,3 \%)]$. Chez la race $T$, c'est au mois de mai qu'elles sont les plus nombreuses $[4,1 \%$ en 1982 $(0$ à $30 \%)$ ]. Cependant à la différence des mâles $V$, on en observe aussi en automne, mais en bien plus faible proportion (fig. 5).

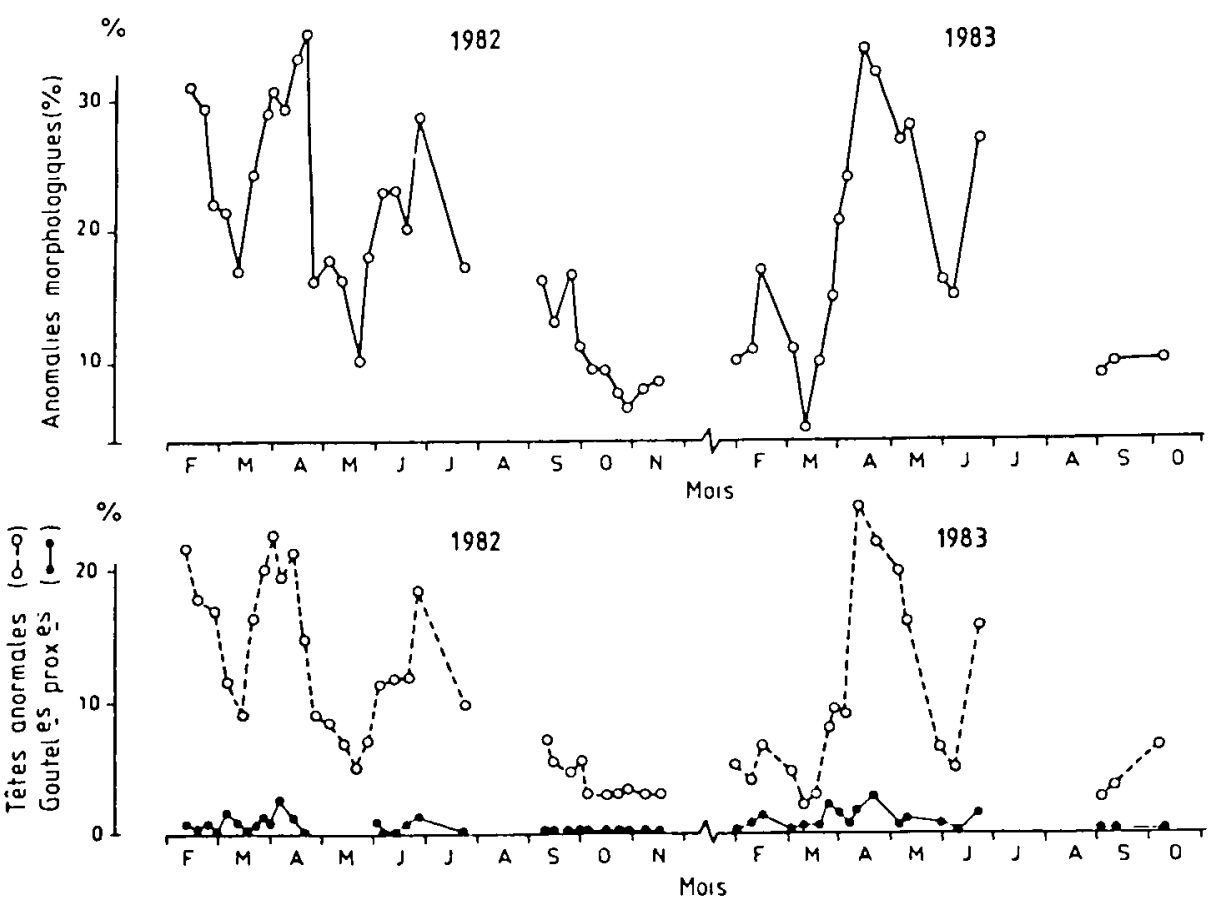

FIG. 4. - Evolution hebdomadaire du pourcentage d'anomalies morphologiques, de têtes anormales et de gouttelettes cytop/asmiques proximales chez le bélier Vendéen, pendant deux années consécutives. 

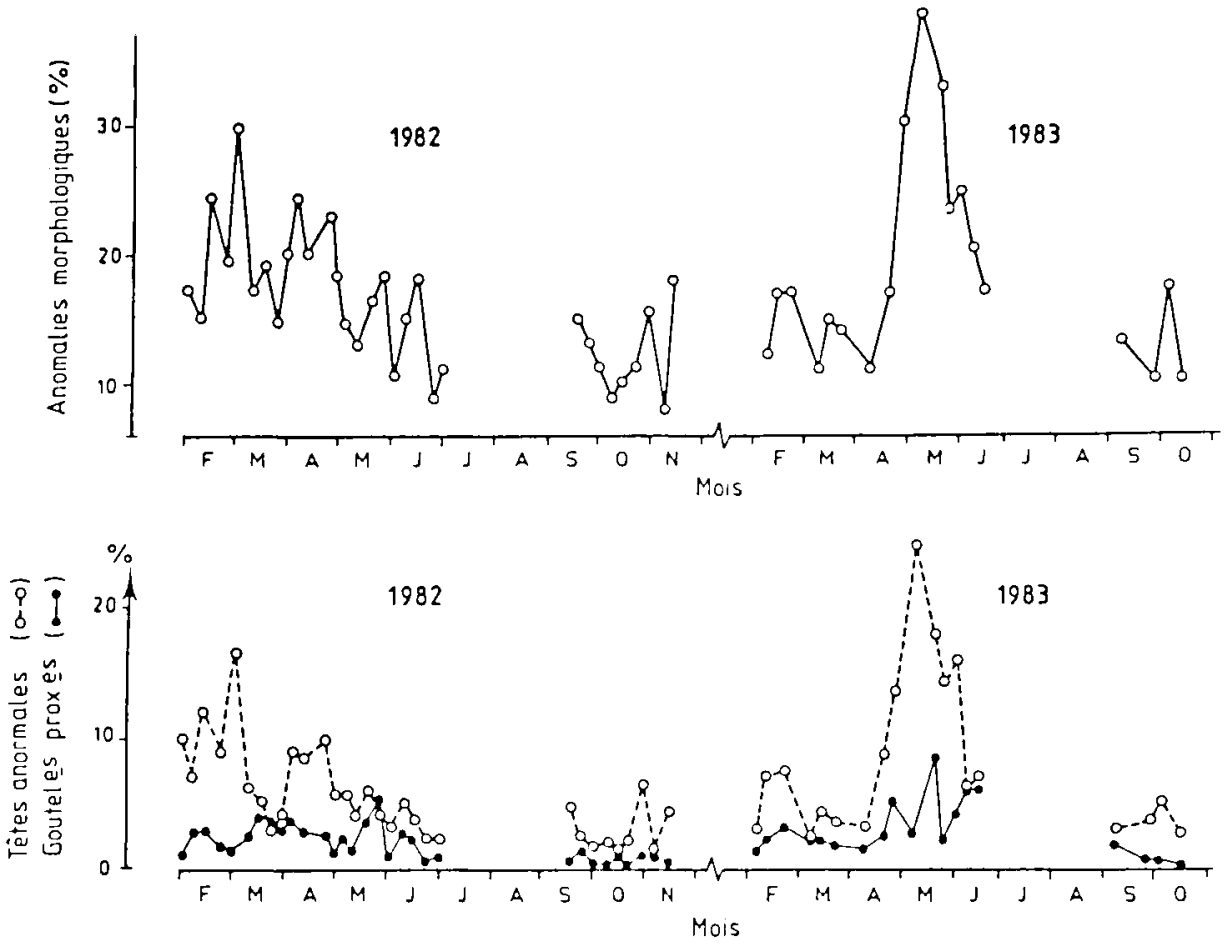

FIG. 5. - Evolution hebdomadaire du pourcentage d'anomalies morphologiques, de têtes anormales et de gouttelettes cytoplasmiques proximales chez le bélier Texel, pendant deux années consécutives.

\section{TABLEAU 1}

Corrélations entre anomalies totales et anomalies de la tête du spermatozoïde chez les beliers Texel et Vendéens.

\begin{tabular}{|c|c|c|c|c|}
\hline \multirow{3}{*}{ Race } & \multicolumn{4}{|c|}{ Année } \\
\hline & \multicolumn{2}{|c|}{1982} & \multicolumn{2}{|c|}{1983} \\
\hline & $P$ & $A$ & $P$ & A \\
\hline Texel & (1) $0,856^{* *}{ }_{1 n}=221$ & $0,785$ (n $=9)$ & $0,96^{* *}(n=15)$ & $0,70_{(n-6)}$ \\
\hline Vendéen & $\begin{array}{l}\text { (1) } 0,917^{* *}(n=20) \\
\text { (2) } 0,952^{* *}(n=20) \\
\text { (3) } 0,885^{* *}(r=20)\end{array}$ & $\begin{array}{l}\left.0,662^{*} \text { (n }=10\right) \\
0,685^{*}(\mathrm{n}=10) \\
0,802^{* *}(\mathrm{n}=10)\end{array}$ & $0,95^{* *}(n=10)$ & non calculé $(n=3$ ) \\
\hline
\end{tabular}

(1) : béliers antenais en $1982 ;(2)$ : béliers adultes ; (3) : tous béliers ; $(1)$ : Couples de moyennes. *: $P<0,05 ;{ }^{*}: P<0,01 ; P$ : printemps; A : Automne. 

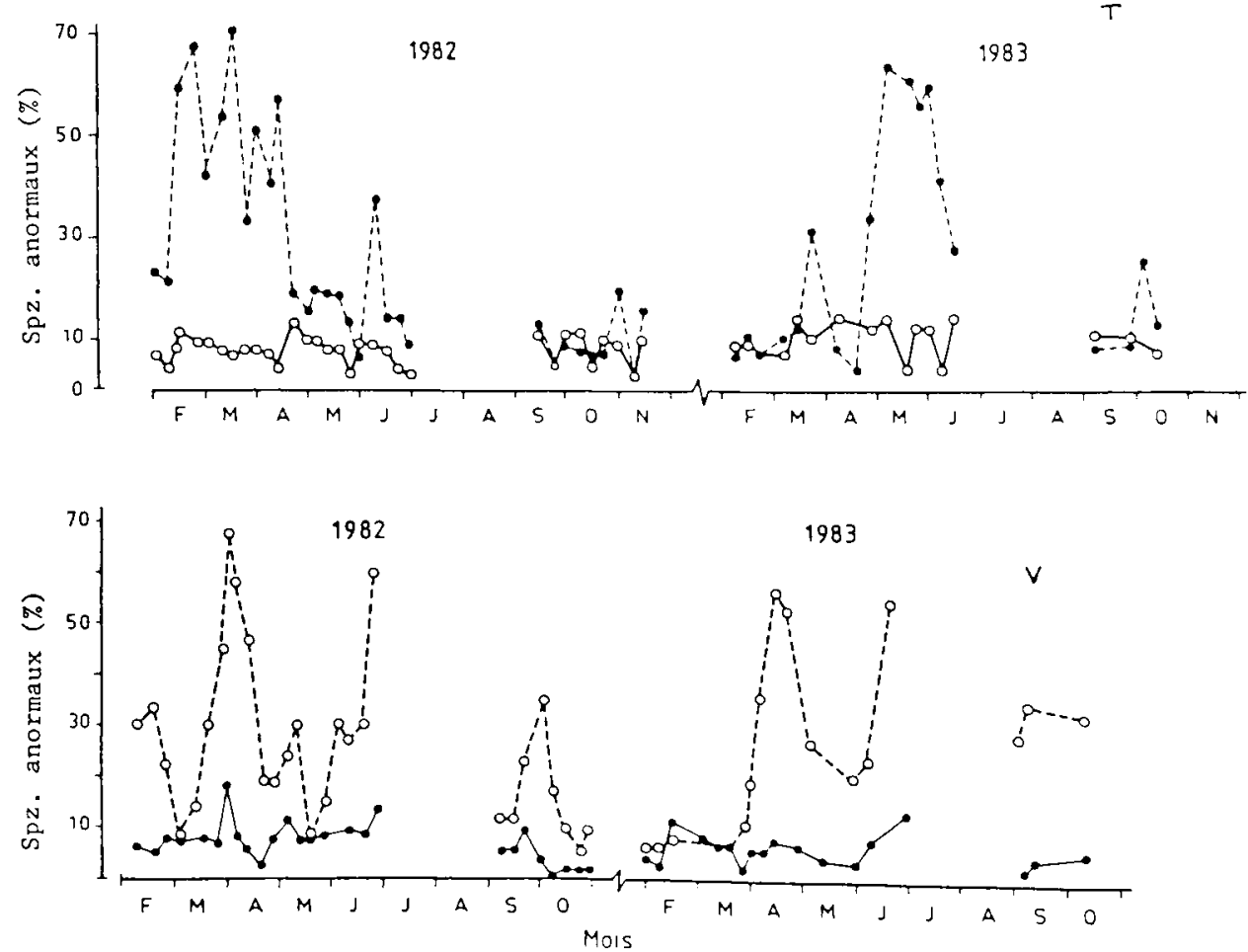

FIG. 6. - Evolution hebdomadaire du pourcentage d'anomalies morphologiques pendant deux années consécutives chez deux béliers Texe/ (T) et chez deux béliers Vendéens (V).

\section{Discussion}

Cette expérience a permis de montrer que le diamètre testiculaire des béliers Texel et Vendéens et la qualité de leurs gamètes sont sujets à de fortes variations au cours de l'année. Le fait de les avoir observées à deux reprises (1982 et 1983) à peu près à la même époque, nous conduit à penser qu'elles sont une des caractéristiques physiologiques du mâle dans ces deux races.

II n'est évidemment pas possible d'affirmer que le seul facteur en cause soit la photopériode puisque dans un milieu naturel, de nombreuses composantes sont susceptibles d'intervenir. Néanmoins, l'importance des phénomènes lumineux dans le contrôle de la fonction de reproduction et les variations qu'ils induisent dans cette espèce (Ortavant et Thibault, 1956 ; Ortavant, 1958, Pelletier, 1971 ; Albério, 1976 ; Schanbacher, 1979 ; Schanbacher et Ford, 1979 ; Lincoln, 1979 ; Colas et al., 1985) nous laissent supposer qu'il existe, chez les deux races, une étroite dépendance de l'activité sexuelle vis-à-vis des conditions naturelles d'éclairement.

a. Poids corporel et diamètre testiculaire. - Le poids corporel des animaux continue d'augmenter jusqu'au mois de mai pour les T et jusqu'au mois d'octobre 
pour les V. Il semble donc que les mâles terminent leur croissance lorsqu'ils ont environ deux ans.

Les mesures testiculaires effectuées sur les béliers $V$ donnent pendant deux années consécutives des résultats comparables : diminution jusqu'à fin mai suivie, aussitôt après, d'une augmentation rapide, décroissance en septembre. Une telle évolution rappelle tout à fait celle que décrit Pelletier (1971) à propos des animaux Ile-de-France.

Chez les sujets $T$, on enregistre bien des moyennes plus élevées en automne qu'au printemps, mais l'allure générale de la courbe est un peu différente d'une année à l'autre notamment en février et en septembre-octobre. Cette différence tient sans doute à l'âge des animaux puisqu'après élimination de l'effet de la croissance corporelle, le diamètre de la gonade est minimum en mai. Toutefois, et c'est là le point essentiel, la croissance testiculaire commence toujours en juin, c'est-à-dire lorsque la durée quotidienne d'éclairement est presque à son apogée.

Ces résultats sont très importants car ils permettent de généraliser la notion de démarrage de l'activité gonadique en jours longs sous nos latitudes. La phase d'étiage se situe donc de février-mars à fin mai.

Il est difficile d'établir une comparaison avec la femelle puisqu'aucune étude précise n'a été réalisée dans les deux races concernées. On sait seulement d'après les travaux de Mauléon et Rougeot (1962), Legan et Karsch (1980), que la reprise de l'activité sexuelle (apparition du premier œestrus) des brebis Limousine, Préalpes ou Suffolk a lieu plusieurs semaines après le passage en jours courts, c'està-dire plus tard que chez les mâles.

Lorsque le poids corporel des animaux s'est stabilisé, on constate (fig. 2) que les dimensions du testicule demeurent en toutes saisons plus élevées chez les béliers $T$ que chez les béliers $V$, bien que ces derniers soient en moyenne plus lourds. On peut donc penser qu'à l'âge adulte, les reproducteurs Texel ont une plus forte production testiculaire ou spermatique, tout au moins en automne lorsque les rendements spermatogénétiques sont voisins de un (Ortavant et Thibault, 1956).

b. Anomalies morphologiques. - Les formes anormales sont, en moyenne, plus abondantes de février à juin que de septembre à octobre. Or, en hiver et au printemps, les températures n'étant pas assez élevées sous nos latitudes pour perturber la fonction de reproduction, la lumière est seule à agir. II semble donc que les jours longs soient au moins en partie à l'origine de cette dégradation de la morphologie des gamètes. Celle-ci a d'ailleurs été signalée à plusieurs reprises dans d'autres races (Fowler, 1965 ; Neves, Günzel et Schmidt, 1980 ; Colas, 1980 ; Mickelsen, Paisley et Dahmen, 1981 ; Amir et al., 1986).

Chez les béliers $v$, les écarts dépendent aussi de l'âge, les sujets antenais étant plus sensibles à l'action de la lumière que les adultes. Cette baisse de qualité maintes fois évoquée à propos de l'agneau (Dunn, 1955 ; Louw et Joubert, 1964 ; Skinner et Rowson, 1968 ; El Wishy, 1974 ; Colas et Zinszner, 1975) n'avait pas encore été observée sur des animaux en fin de croissance.

II est intéressant de souligner que les valeurs maximales observées en 1982 et 1983 sont identiques et apparaissent dans le sperme toujours à la même époque. 
Le profil morphologique que nous avons décrit peut donc être considéré comme caractéristique de cette race.

Au mois de juin, on assiste à une nouvelle dégradation de la semence (V). II s'agit essentiellement d'anomalies de la tête des spermatozoïdes, qui ont dû prendre naissance au cours de la spermiogenèse ou de la maturation épididymaire. L'existence de telles malformations est difficile à expliquer a priori. Peut-être estelle liée à une déficience en testostérone. On sait, en effet, que les processus de maturation des gamètes dépendent des androgènes (Orgebin-Crist, Nader et Hoffman, 1976). Un dosage de l'hormone plasmatique nous aurait sans doute éclairés sur ce sujet.

Lorsque les jours rallongent, la qualité des éjaculats diminue beaucoup chez les béliers Texel. Ceux-ci sont donc également sensibles à l'environnement lumineux. Toutefois, les phénomènes se répètent moins d'une expérience à l'autre. Ils sont également moins marqués et on n'enregistre pas d'élévation anormale du taux d'A.M. au mois de juin.

Il existe, au printemps, dans les deux races, d'énormes différences individuelles dans les pourcentages maxima d'A.M. Certains animaux antenais ou adultes possèdent parfois une ou plusieurs malformations sur la quasi totalité de leurs spermatozoïdes. D'autres, au contraire, présentent toute l'année peu de formes anormales. D'où l'intérêt de procéder à un tri sévère des reproducteurs ou des éjaculats avant leur mise en service. Nous avons pu vérifier sur des béliers $T$, différents de ceux de notre expérience, que le taux de fertilité pouvait varier de 1 à 3,5 (26,7 à $82,7 \% ; \mathrm{P}<0,01$; résultats non publiés) selon la qualité initiale du sperme.

Les défauts situés au niveau de la tête de la cellule sont à cette époque étroitement corrélés à l'ensemble des anomalies. Leur fréquence parfois supérieure à $20 \%$ donne une idée des perturbations que les phénomènes lumineux sont susceptibles de provoquer dans les processus spermatogénétiques. Elle laisse également présager une diminution de la fertilité, puisqu'il a été montré, chez plusieurs espèces (Rathore, 1968 ; David et al., 1972 ; Sauvalle et al., 1976), que les malformations de la tête sont impliquées dans la baisse de fécondance d'un éjaculat.

Les gouttelettes cytoplasmiques en position proximale n'apparaissent qu'au printemps dans le sperme des béliers Vendéens et en faible proportion. Chez les bélier Texel, la fréquence est un peu plus élevée et quelques éjaculats en renferment encore en automne. II semble donc que pour certains animaux, la maturation épididymaire reste perturbée même en saison sexuelle (Fournier-Delpech et al., 1979).

\section{Conclusion}

Les races Vendéenne et Texel connaissent de fortes variations d'activité sexuelle au cours de l'année. Le diamètre testiculaire et le pourcentage de spermatozoïdes normaux sont plus faibles de février à juin que de septembre à octobre ou novembre. 
La reprise de la croissance testiculaire se produit toujours en juin, c'est-à-dire en jours longs quelle que soit la race ou l'année. La période d'activité minimale se situe donc de février-mars à fin mai.

II existe d'énormes différences individuelles au printemps en ce qui concerne les anomalies morphologiques. Celles-ci s'estompent en automne.

Les anomalies de la tête des spermatozoïdes sont hautement corrélées aux anomalies totales de février à juin.

Recu en décembre 1985. Accepté en avril 1986.

Remerciements. - Les auteurs tiennent à remercier Monsieur R. Ortavant pour ses commentaires et le secrétariat qui a assuré la frappe du texte.

\section{Références}

ALBÉRIO R., 1976. Rôle de la photopériode dans le développement de la fonction de reproduction chez l'agneau lle-de-France, de la naissance à 21 mois. Th. Doct. Univ. Paris.

AMIR D., GACITUA H., RON M., LEHRER A. R., 1986. Seasonal variation in semen characteristics and the fertility of Finn Cross rams subjected to frequent ejaculations. Anim. Reprod. Sci., 10, 75-84.

COLAS G., 1980. Variations saisonnières de la qualité du sperme chez le bélier lle-de-France. 1. Etude de la morphologie cellulaire et de la motilité massale. Reprod. Nutr. Dévelop., 20, 1789 . 1799.

COLAS G., 1981. Variations saisonnières de la qualité du sperme chez le bélier lle-de-France. II. Fécondance : relation avec les critères qualitatifs observés in vitro. Reprod. Nutr. Dévelop., 21. 399-407.

COLAS G., ZINSZNER F., 1975. Production spermatique et développement testiculaire chez l'agneau de race Ile-de-France et Préalpes. $1^{\text {ères }}$ Journées de la Recherche ovine et caprine, "Les races prolifiques ». ITOVIC édit. 235-243.

COLAS G., GUÉRIN Y., CLANET V., SOLARI A., 1985. Influence de la durée d'éclairement sur la production et la fécondance des spermatozoïdes chez le bélier adulte lle-de-France. Reprod. Nutr. Dévelop., 25, 101-111.

COLYER R. J., 1971. Development of the testis and epididymis of the Clun Forest ram. J. agric. Sci. Camb., 76, 433-441.

COUROT M., 1971. Etablissement de la spermatogenèse chez l'agneau (ovis aries). Etude expérimentale de son contrôle gonadotrope : importance des cellules de la lignée sertolienne. Th. Doct. ès Sci. nat. Univ. Paris IV, A.0.6317. 200 p.

DAVID G., BISSON J. P., JOUANNET P., CZYGLIK F., GERNIGON C., ALEXANDRE C., DREYFUS G., 1972. Les tératospermies, 81-102. Dans Fécondité et stérilité du mâle. Acquisitions récentes. Masson Ed., Paris.

DUNN R. B., 1955. Puberty in Merino rams. Austr. J. agric. Res., 11, 805-826.

EL WISHY A. B., 1974. Some aspects of reproduction in fattailed sheep in subtropics. IV. Puberty and sexual maturity. Tierz. Züchtungsbiol., 91, 311-316.

FOURNIER-DELPECH S., COLAS G., COUROT M., ORTAVANT R., BRICE G., 1979. Epididymal sperm maturation in the ram : motility, fertilizing ability and embryonic survival after uterine insemination in the ewe. Ann. Biol. anim. Bioch. Bioph., 19, 597-605.

FOWLER D. G., 1965. Semen quality of Merino rams. 2. The effects of seasonal changes in daylength on semen quality. Austr. J. exp. Agric. anim. Husb., 5, 247-251.

LEGAN S. J., KARSCH F. J., 1980. Photoperiodic control of seasonal breeding in ewes : modulation of the negative feedback action of estradiol. Biol. Reprod., 23, 1061-1068. 
LINCOLN G. A., 1979. Photoperiodic control of seasonal breeding in the ram : participation of the cranial sympathetic nervous system. J. Endocr., 82, 135-147.

LOUW D. F., JOUBERT D. M., 1964. Puberty in the male Dorper sheep and Boer goat. South Afric. J. agric. Sci., 7, 509-520.

MAULÉN P., ROUGEOT J., 1962. Régulation des saisons sexuelles chez des brebis de races différentes au moyen de divers rythmes lumineux. Ann. Biol. anim. Bioch. Biophys., 2, 209. 222.

MICKELSEN W. D., PAISLEY L. G., DAHMEN J. J., 1981. The effect of season on the scrotal circumference and sperm motility and morphology in rams. Theriogenology, 16, 45-51.

NAGASE N., GRAHAM E. F., 1964. Pelleted semen : comparison of different extenders and processes on fertility of bovine spermatozoa. Proc. 5th int. Congr. anim. Reprod. artif. Insem., Trento, 2, 387-389.

NEVES J. P., GÜNZEL A. R., SCHMIDT H., 1980. Einfluss der Jahreszeit auf der Sperma Qualität von Merino Fleischschafen. Zuchthygiene, 15, 118-125.

ORGEBIN-CRIST M. C., NADER J., HOFFMAN L. H., 1976. The effects of testosterone, $5 \alpha$-dihydrotestosterone, $3 \alpha$-androstanediol and $3 \beta$-androstanediol on the maturation of rabbit epididymal spermatozoa in organ culture. Cell Tiss. Res., 167, 515-525.

ORTAVANT R., 1958. Le cycle spermatogénétique chez le belier. Th. Doct. Sci., Paris, 127 pp., $N^{\circ}$ A 3118-0 3990.

ORTAVANT R., THIBAULT C., 1956. Influence de la durée d'éclairement sur la production spermatique du bélier. C.R. Soc. Biol., 150, 358-361.

PELLETIER J., 1971. Influence du photopériodisme et des androgènes sur la synthèse et la libération de $\angle H$ chez le belier. Th. Doct. Etat ès Sci. nat. Paris $N^{\circ}$ A 5441, p. 243.

RATHORE A. K., 1968. Effect of high temperature on sperm morphology and subsequent fertility in Merino sheep. Proc. austr. soc. anim. Prod., 7, 270-274.

SAUVALlE A., HERLICOVIEZ D., DESHAYES M., TZARD J., 1976. Analyse de 144 spermocytogrammes. Arch. Anat. Cytol. Path., 24, 369-377.

SCHANBACHER B. D., 1979. Increased lamb production with rams exposed to short daylengths during the non-breeding season. J. anim. Sci., 49, 927-932.

SCHANBACHER B. D., FORD J. J., 1979. Photoperiodic regulation of ovine spermatogenesis: relationship to serum hormones. Biol. Reprod., 20, 719-726.

SKINNER J. D., ROWSON L. E. A., 1968. Puberty in Suffolk and crossbred rams. J. Reprod. Fert., 16, 479-488.

WIGGINS E. L., TERRILL C. E., EMIK L. D., 1953. Relationships between libido, semen characteristics and fertility in range rams. J. anim. Sci, 12, 684-696. 